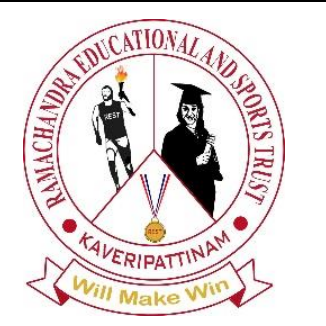

REST Journal on Emerging trends in Modelling and Manufacturing

Vol: 6(3), 2020

REST Publisher

ISSN: 2455-4537

Website: www.restpublisher.com/journals/jemm

\title{
A Study on Modelling of Flexible Manufacturing System
}

\author{
Muhammed Zakariya Wagadiya, Kavya Gupta,
}

MPSTME, SVKM'S NMIMS University, Shirpur, Dhule, Maharashtra, India

\begin{abstract}
Flexible Manufacturing System (FMS) is flexible in nature it helps in the automatic production of the products in mid-level range, it can also be called as automatic production system. In this paper we are discussing about Flexible Manufacturing System, Petri Nets, Scheduling, Analytical Model, Mathematical Model, Performance Evaluation. FMS is a manufacturing method which adapt to the quality and quantity or the type product which is to be manufactured. A petri Nets directed bipartite graph in which notes represent transition and places. In Scheduling tool loading problem and scheduling in flexible manufacturing system are considered simultaneously. In Analytical Model, are also called mathematical model in which a number of directions in which models required extension are outlined. In Mathematical model is a description of a system using mathematical concepts to construct a mathematical model which is also called mathematical modelling. In Performance Evaluation, in this performance is evaluated of a flexible manufacturing system, and the type of technique used are queueing network, state allocation, simulation etc.
\end{abstract}

\section{Introduction}

Flexible Manufacture System in the present investigation, a queueing model is developed to predict the performance of the FMS with a multiple discrete material handling devices(MHD). An iterative method has been suggested in which two queueing network model are considered to determine the MHD interference using MVA for state dependent routing [2]. Flexible manufacturing systems represent an advanced class of automated manufacturing systems. Because of the complexity and dynamic behaviour of such systems, simulation modelling is becoming a popular method of facilitating FMS design and determining operational strategies. This paper illustrates the use of activity cycle diagrams as a graphical method for developing simulation models for two existing flexible manufacturing systems [4]. Scrubbed introduced many numerous extension and refinements to event graphs for simulation modelling. The results obtained are integrated with Scrubbed work for complete description of event graphs and analysis. The modelling of FMS can be illustrated by event graphs modelling and analysis [5]. A powerful tool is used for analysing asynchronous concurrent system known as Petri Nets. The formulation for developing of modular models of FMS is done by Petri Nets which is shown in this paper. The model whose resources are shared, constraint of the product to be fabricated in the flexible manufacturing system is done by coloured timed extended Petri Nets [18]. FMS has been brought to many manufacturing industries due to the global market competition. As this technology advancement has brought various success to the industries which varies in technology application. Cast in a fractal architecture, the model of FMS admits a natural hierarchical decomposition of highly decoupled units with similar structure and control. As a consequence, this model manages the structural complexity and coordination of an FMS hierarchy by maximizing local functionality and minimizing global control [11]. The paper on flexible manufacturing system: A review on analytical model has been helped in the development of analytical model in FMS. A summarized and an assessment in the strength and weaknesses of modelling approach has been of each group contributions for the model development [12]. The challenges inflicted by the global market, ever changing customer demands, rapid delivery of product to market and advancement in technology has forced manufacturing industry to adapt flexible manufacturing system. There are enablers which helps in the transition process from traditional manufacturing system to FMS and also in the implementation of FMS in the manufacturing industry. [13]. The PNs which are more suitable for modelling and simulation of FMS knows as the new extended stochastic high level evaluation petri nets (ESHLEP-N) is introduced in this paper. In ESHLEP-N the resulting model are simpler, more intuitive and more descriptive, and also have decisive power. The scheduling and simulation expert system for flexible manufacturing system is designed and established on the basis of ESHLEP-N model [15]. Flexible manufacturing systems (FMSs) are a class of automated systems which can be used to improve productivity in discrete parts manufacturing. Due to the complexity of these systems, it is useful to have models that can aid in the design and operation of FMSs. We specifically differentiate here between generative (or prescriptive) models, which find candidate decisions, and evaluative (or descriptive) models, which evaluate a given set of decisions [18]. This paper shows the steps how the planning and scheduling problem of FMS are solved simultaneously using hierarchical process. The three most important FMS planning problems are integrating and formulate batching, loading and routeing. According to the optimal decisions provided by the integrated planning model, to generate detail parts sequencing in the sequence independent environment we use offline 
scheduling scheme [23]. Petri Net in Dynamic system to exhibit the modelling asynchronous discrete events coloured petri nets(CPNs) have been used. While for approximate reasoning and uncertainty knowledge inference Fuzzy Petri Nets (FPNs) have been used. While to overcome the modelling of both dynamic system and inexact production inference of FMS a new net base structure is proposed is called as Fuzzy Coloured Petri Nets(FCPNs) [32]. The application of an algebraic representation for a restricted class of decision-free Petri nets to a typical flexible manufacturing system (FMS) problem is considered. A new mapping is introduced to the complex number field for the time-event information-states, which allows the algebraic representation to be readily manipulated numerically. The utility of this approach is illustrated with the MATLAB package and applied to an example [33]. The system which has computer integrated systems, which have many concurrent components, very complicated logical relations and a distributed computer system structure is knowns as FMS. This system has been extensively industry. Furthermore, both classical and extended for timing analysis, PNs definition are reviewed in this paper. In recent research a review is done in which PN theory is applied to the design and analysis of flexible manufacturing system [34]. By using the extension of PNs formalism an issue is subjected on the modelling of concurrent system structure, is addressed in this paper. In FMS model we have applied the concept of reconfigurable finite capacity PNs [42].

\section{Queueing networks}

The first step is to interference from the material handling device by inflating the station service times but neglect queueing at the material handling device, while in another network, the queueing for the MHD is taken into consideration. By iterating the two networks we can obtained the performance of flexible manufacturing system. We obtained better results from algorithm which were suggested rather than from the earlier workers for single MHD [3]. This paper outlines the state-ofthe-art in studying flexible manufacturing systems (FMSs) using analytical queueing network models. These include Jackson networks, reversible networks and approximate models of non-product-form networks. The focus is on identifying the major features of the models as they relate to the operational characteristics of FMSs. Prescriptive models concerning the optimal design and/or operational control of FMS networks are also discussed [25]. We consider flexible manufacturing systems (FMSs) which are composed of a set of workstations linked together with a material handling system (MHS). Each workstation consists of an input buffer, a single machine and an output buffer. The MHS consisting of a single cart routes work among the workstations according to the process paths required by the work. We deal with an optimal control problem in this FMS. We model the FMS as a closed queueing network [21].

\section{Scheduling}

In Scheduling process for the designing and operations of complex system, it is necessary to have tools which predict the performance of system under various conditions. Scheduling is necessary in FMS for automated manufacturing of parts with medium in demand [1]. The tool loading problem is simultaneously considered in FMS Ian this research. Many parallel CNC machines are used to process various jobs. A set of machine tools are required to process each job, due to the economic limitations many copied tools are not available. [45].

\section{Analytical Model}

A number of directions in which models require extension are outlined, in particular the representation of such aspects of FMS operation as the tool delivery systems, the blocking phenomenon, the transient behaviour and the differences between flexible machining systems and flexible assembly systems. Further work is also required on the structure of FMS control and the integration with plant production planning and control. [12]. Besides providing the designer a facile means of expressing the dynamic character of the flexible manufacturing system, the formalism also simplifies the model. In the second part of the paper we present and also compare the Petri net model and the equivalent reconfigurable finite capacity Petri net model for a such a system and we evaluate these models using PetriNetExec, a software library supporting the integration of Petri nets into Java applications [42].

\section{Mathematical model}

The ability of being flexible in nature FMS also help with automatic production of different parts in mid-range. It can also be known as automatic manufacturing system. The research and development of FMS modelling is being provided in this paper for the better understanding of flexible manufacturing system. Different modelling techniques were used in FMS like: - mathematical, artificial intelligence, hierarchical, multi criteria decision making method, PNs and simulation. The investigation work of FMS was accomplished by using these techniques [7]. The type of problem faced in FMS is demonstrated on the application of multi-faceted modelling. In this combination of Petri nets is used for high-level abstraction of the controlled processes. With the help of this model we can analyse system performance. To analyse it we need it minimize the algebraic representation for restricted decision free PNs as it is used for modelling the mechanism to combine both abstraction levels to produce multi modelled FMS [33].

\section{Performance evaluation}

This paper cover in practical manner about the pros and cons of the two classes of model. In Flexible manufacturing system, evaluate models are divided into five different types of classes, the techniques used are: - Static Allocation, network theory, simulation, perturbation analysis, and petri. The advantage and disadvantages are being briefly described in this paper [18]. In the designing and operation of flexible manufacturing system performance evaluation and modelling has played a vital 
role. In the recent contributions three stochastic modelling techniques are represented for the modelling of FMS are Markov and semi-Markov Processes, queueing networks and stochastic Petri Nets, the major role of these techniques is to construct simple, illustrative FMS example [36].

\section{Conclusion}

Flexible Manufacturing System is an advance class of automated Manufacturing system, as simulation modelling is a popular method of facilitating flexible manufacturing System design and determining operational strategies because of complexity and diversity of system. Petri Nets(PNs) have been extensively used modelling of asynchronous discrete events exhibited in dynamic system. Fuzzy Petri Nets have been used for the system which involves inference of approximate reasoning and uncertainty knowledge. In Queueing network, we model the interference by inflating the station service by MHD but neglecting the queue MHD. We can have obtained the performance of FMS configuration by iterating between two networks. The Scheduling and tool loading problem is considered simultaneously in flexible manufacturing system. There are various jobs that must be processed on a number of parallel computer numerical control machines. The processing of each job requires a set of machine tools. In Analytical model models require extensions are outlined in number of directions for representation in particular aspects of FMS operation as the tool delivery system the blocking phenomenon, the transient behaviour and the differences between flexible machining systems and flexible assembly systems. In Mathematical model a minimax algebraic representation for restricted decision-free Petri nets is used as the modelling mechanism to combine both abstraction levels to produce a "multi-model" of the FMS.

\section{References}

[1].Suri, Rajang, and Richard R. Hildebrant. "Modelling flexible manufacturing systems using mean-value analysis." Journal of manufacturing systems 3, no. 1 (1984): 27-38.

[2].Narahari, Y., and N. Viswanadham. "A Petri net approach to the modelling and analysis of flexible manufacturing systems." Annals of operations research 3, no. 8 (1985): 449-472.

[3].Jain, M., Sandhya Maheshwari, and K. P. S. Baghel. "Queueing network modelling of flexible manufacturing system using mean value analysis." Applied Mathematical Modelling 32, no. 5 (2008): 700-711.

[4].Hlupic, Vlatka, and Ray J. Paul. "Simulation modelling of flexible manufacturing systems using activity cycle diagrams." Journal of the Operational Research Society 45, no. 9 (1994): 1011-1023.

[5].Sargent, Robert G. "Event graph modelling for simulation with an application to flexible manufacturing systems." Management science 34, no. 10 (1988): 1231-1251.

[6].Ramanan, G., J. Edwin Raja Dhas, and M. Ramachandran. "Optimization of Material Removal Rate and Surface Roughness for Wire Electric Discharge Machining of AA7075 Composites using Grey Relational Analysis." International Journal of Vehicle Structures and Systems 9, no. 5 (2017).

[7].Suri, Rajan. "An overview of evaluative models for flexible manufacturing systems." Annals of Operations Research 3, no. 1 (1985): 13-21.

[8].Pramod Raichurkar, M. Ramachandran, V. Subramaniam, performance of silk yarn with the effect of soaking in mrudula soaking oil, colourage 64(1):41-44, 2017

[9].Chen, Injazz J., and Chia-Shin Chung. "Sequential modelling of the planning and scheduling problems of flexible manufacturing systems." Journal of the Operational Research Society 47, no. 10 (1996): 1216-1227.

[10].Veung, D. S., James NK Liu, Simon CK Shiu, and George SK Fung. "Fuzzy coloured Petri nets in modelling flexible manufacturing systems." In Proceedings Mexico-USA Collaboration in Intelligent Systems Technologies., pp. $100-107$. IEEE, 1996.

[11].Bundell, Gary. "Multi-faceted modelling of Flexible Manufacturing Systems using a Minimax Algebraic Petri net representation." In Proceedings of IEEE 2nd International Workshop on Emerging Technologies and Factory Automation (ETFA'93), pp. 177-187. IEEE, 1993.

[12].Narahari, Y., and N. Viswanadham. "Performance Modelling of Flexible Manufacturing Systems." IETE Journal of Research 35, no. 4 (1989): 221-236.

[13].Târnaucă, Bogdan, Dan Puiu, Vasile Comnac, and Constantin Suciu. "Modelling a flexible manufacturing system using reconfigurable finite capacity Petri nets." In 2012 13th International Conference on Optimization of Electrical and Electronic Equipment (OPTIM), pp. 1079-1084. IEEE, 2012.

[14].Zhou, MengChu, and Frank DiCesare. "Petri net modelling of buffers in automated manufacturing systems." IEEE Transactions on Systems, Man, and Cybernetics, Part B (Cybernetics) 26, no. 1 (1996): 157-164.

[15].Özpeynirci, Selin. "A heuristic approach based on time-indexed modelling for scheduling and tool loading in flexible manufacturing systems." The International Journal of Advanced Manufacturing Technology 77, no. 5-8 (2015): 1269-1274. [16].Nishita bhise, M. Ramachandran, Design and Numerical evaluation of crankshaft of diesel engine for total deformation and strain IOP Conference Series: Material Science and Engineering, 810 (2020) 012010. 